\title{
The cAMP-PKA Pathway Regulates Growth, Sexual and Asexual Differentiation, and Pathogenesis in Fusarium graminearum
}

\author{
Shuai Hu, ${ }^{1}$ Xiaoying Zhou, ${ }^{2}$ Xiaoying Gu, ${ }^{1}$ Shulin Cao, ${ }^{1}$ Chengfang Wang, ${ }^{1}$ and Jin-Rong Xu ${ }^{2}$ \\ ${ }^{1}$ State Key Laboratory of Crop Stress Biology for Arid Areas, College of Plant Protection, Northwest A\&F University, \\ Yangling, Shanxi, China; ${ }^{2}$ Department of Botany and Plant Pathology, Purdue University, West Lafayette, IN, U.S.A.
}

Submitted 10 October 2013. Accepted 14 January 2014.

\begin{abstract}
Like many other filamentous ascomycetes, Fusarium graminearum contains two genes named $C P K 1$ and $C P K 2$ that encode the catalytic subunits of cyclic AMP (cAMP)-dependent protein kinase A (PKA). To determine the role of cAMP signaling in pathogenesis and development in $F$. graminearum, we functionally characterized these two genes. In addition, we generated and characterized the cpk1 cpk2 double and $f a c 1$ adenylate cyclase gene deletion mutants. The $c p k 1$ mutant was significantly reduced in vegetative growth, conidiation, and deoxynivalenol production but it had increased tolerance to elevated temperatures. It was defective in the production of penetration branches on plant surfaces, colonization of wheat rachises, and spreading in flowering wheat heads. Deletion of CPK1 had no effect on perithecium development but the $c p k 1$ mutant was defective in ascospore maturation and releasing. In contrast, the $c p k 2$ mutant had no detectable phenotypes, suggesting that $C P K 2$ contributes minimally to PKA activities in $F$. graminearum. Nevertheless, the cpk1 cpk2 double mutant had more severe defects in vegetative growth and rarely produced morphologically abnormal conidia. The double mutant, unlike the cpk1 or cpk2 mutant, was nonpathogenic and failed to form perithecia on self-mating plates. Therefore, $C P K 1$ and $C P K 2$ must have overlapping functions in vegetative growth, differentiation, and plant infection in $F$. graminearum. The fac 1 mutant was also nonpathogenic and had growth defects similar to those of the cpk1 cpk2 mutant. However, deletion of FAC1 had no effect on conidium morphology. These results indicated that $C P K 1$ is the major PKA catalytic subunit gene and that the cAMP-PKA pathway plays critical roles in hyphal growth, conidiation, ascosporogenesis, and plant infection in $F$. graminearum.
\end{abstract}

In many plant-pathogenic fungi, the cyclic AMP (cAMP)dependent signaling pathway is one of the major signal transduction pathways that regulates various differentiation and infection processes (Brakhage and Liebmann 2005; Choi and Xu 2010; Lee et al. 2003; Lengeler et al. 2000; Li et al. 2012). The cAMP-dependent protein kinase A (PKA) is the major

Corresponding authors: C. Wang; E-mail: wangchenfang@nwsuaf.edu.cn; Telephone: +86-029-87081270; and J.-R. Xu; E-mail:jinrong@purdue.edu

* The $\boldsymbol{e}$-Xtra logo stands for "electronic extra" and indicates that four supplementary figures and one supplementary table are published online.

(C) 2014 The American Phytopathological Society downstream effector of the cAMP signaling pathway. In the absence of CAMP, a secondary messenger synthesized by adenylate cyclase, the PKA holoenzyme exists as an inactive heterotetramer composed of two regulatory $(\mathrm{R})$ subunits and two catalytic (C) subunits. Binding of cAMP to R subunits leads to the activation of two monomeric $\mathrm{C}$ subunits, which can phosphorylate downstream target proteins and regulate a variety of physiological processes.

Filamentous fungi normally have two genes encoding the catalytic subunits of PKA and one of them often is responsible for most of the PKA activities (Banno et al. 2005; Lee et al. 2003; Ni et al. 2005; Schumacher et al. 2008). In a number of them, deletion of the major PKA catalytic subunit gene, such as FoCPKA of Fusarium oxysporum, CPK1 of $F$. verticillioides, and $P K A C-1$ of Neurospora crassa, results in reduced growth rate and conidiation, which is similar to deletion of the adenylate cyclase gene (Choi and Xu 2010; Kim et al. 2011; Shimizu and Keller 2001). However, deletion of the PKA catalytic subunit gene $C P K A$ or adenylate cyclase gene $M A C 1$ in Magnaporthe oryzae had no significant effect on growth rate, although the $c p k A$ mutant was delayed in appressorium formation and defective in appressorium turgor generation (Adachi and Hamer 1998; Choi and Dean 1997; Mitchell and Dean 1995). In Botrytis cinerea, conidiation was not impaired in the $\Delta$ bcpkal mutant (Schumacher et al. 2008). Interestingly, conidiation is de-repressed in the $c r-1$ adenylate cyclase mutant in $N$. crassa (Banno et al. 2005). In Aspergillus nidulans, the pkaA gene also negatively regulates asexual sporulation (Shimizu and Keller 2001).

In plant- and human-pathogenic fungi, cAMP signaling also is known to play a species-specific role in regulating sexual reproduction, pathogenesis, and other biological processes (Lee et al. 2003; Lengeler et al. 2000). For example, in Ustilago maydis, the adenylate cyclase mutant and the PKA catalytic subunit mutant grew similarly to the wild-type but were defective in pathogenesis on maize (Durrenberger et al. 1998). In Cryptococcus neoformans, the cacl adenylyl cyclase mutant was defective in mating hypha production and failed to produce capsule and melanin (Alspaugh et al. 2002). Similar to the $c p k A$ or $m a c l$ mutant in M. oryzae, mutants deleted of a PKA catalytic subunit gene or the adenylate cyclase gene in Colletotrichum lagenarium and $C$. trifolii were nonpathogenic and formed nonfunctional appressoria (Yamauchi et al. 2004; Yang and Dickman 1999). In Alternaria alternata, the $\Delta a a p k 1$ mutant was reduced in Alternaria alternata toxin production and virulence (Xu et al. 2011). In Verticillium dahlia, PKA regulates ethylene biosynthesis, which is directly related to virulence (Tzima et al. 2010). In B. cinerea, the adenylate 
cyclase and PKA catalytic subunit genes are important for plant infection but deletion of these genes has no effect on the production of oxalic acid and botrydial, two important virulence factors (Schumacher et al. 2008).

$F$. graminearum is a major causal agent of wheat and barley head blight or scab worldwide (Bai and Shaner 2004). Fusarium head blight (FHB) poses a serious problem in wheat production by causing severe yield losses and contamination of infested kernels with harmful mycotoxins, including deoxynivalenol (DON) and zearalenone (Desjardins 2003; Goswami and Kistler 2004). In a previous study of systematic characterization of the $F$. graminearum kinome, we identified the mutants deleted of two PKA catalytic subunit genes named $C P K 1$ and $C P K 2$. To better understand the cAMP-PKA pathway in $F$. graminearum, in this study, we further characterized the $c p k 1$ and $c p k 2$ mutants. In addition, we generated and characterized the $c p k 1$ cpk 2 double and facl adenylate cyclase gene deletion mutants. Whereas the cpk2 mutant had no detectable phenotypes, the cpkl mutant had pleiotropic defects in growth, conidiation, DON production, and ascospore maturation. It was defective in the production of penetration branches on plant surfaces and colonization of wheat rachises. Although deletion of both PKA catalytic subunit genes is lethal in Aspergillus nidulans (Ni et al. 2005), the cpkl cpk2 double mutant in $F$. graminearum was viable, although it had more severe defects than the cpkl mutant and was sterile in self-crosses and nonpathogenic in infection assays. The facl mutant had defects similar to those of the cpk1 cpk2 double mutant in vegetative growth and pathogenesis but it still produced normal conidia. Overall, our data indicate that $C P K 1$ is the major PKA catalytic subunit in $F$. graminearum but $C P K 1$ and $C P K 2$ have overlapping functions. The cAMP-PKA pathway plays critical roles in hyphal growth, conidiation, ascosporogenesis, and plant infection in F. graminearum.

\section{RESULTS}

\section{$C P K 2$ but not $C P K 1$ is dispensable for growth and conidiation.}

The genome of $F$. graminearum contains two PKA catalytic subunit genes, FGSG_07251 and FGSG_08729, that were named $C P K 1$ and $C P K 2$, respectively, in this study. Phylogenetic analysis revealed that these two genes are well conserved in other filamentous fungi (Supplementary Fig. S1). CPK1 is orthologous to yeast $T P K 2, C P K A$ of $M$. oryzae (Mitchell and Dean 1995), and $a d r l$ of $U$. maydis (Durrenberger et al. 1998). The cpk1 and cpk2 mutants (Table 1) were generated in a previous study of the $F$. graminearum kinome (Wang et al. 2011). Whereas the $c p k 2$ mutant had no detectable phenotype, the cpk1 mutant C1M-1 was significantly reduced in growth rate (Fig. 1A). Colonies formed by the cpkl mutant had limited aerial hyphal growth.

In comparison with the wild type, the cpkl mutant was reduced approximately $44 \%$ in conidiation in carboxy-methyl cellulose (CMC) cultures, which is not proportional to its tenfold reduction in growth rate (Table 2). Microscopic examination revealed that the $c p k 1$ mutant tended to produce phialides and conidia earlier than the wild type. After incubation for $16 \mathrm{~h}$, phialides and conidia were observed in CMC cultures of mutant $\mathrm{C} 1 \mathrm{M}-1$. Under the same conidiation, phialides were not produced by the wild-type strain PH-1 (Fig. 1B). It is likely that the cpkl mutant was defective in nutrient sensing and derepressed in asexual reproduction, which may be related to its reduced vegetative growth. Although only data for the $c p k 1$ mutant C1M-1 and cpk2 mutant C2M-1were presented, mutants C1M-3 and C1M-6 and mutants C2M-8 and C2M-14 had phenotypes similar to $\mathrm{C} 1 \mathrm{M}-1$ and $\mathrm{C} 2 \mathrm{M}-1$, respectively.

For complementation assays, the wild-type $C P K 1$ allele was reintroduced into the cpkl mutant C1M-1. Defects in hyphal growth and other phenotypes described below were rescued in the resulting cpk1/CPK1 transformant C1C-1 (Table 1). These results indicate that deletion of $C P K 1$ is directly responsible for the defects observed in the cpkl mutant and $C P K 1$ is important for normal vegetative growth and conidiation in $F$. graminearum.

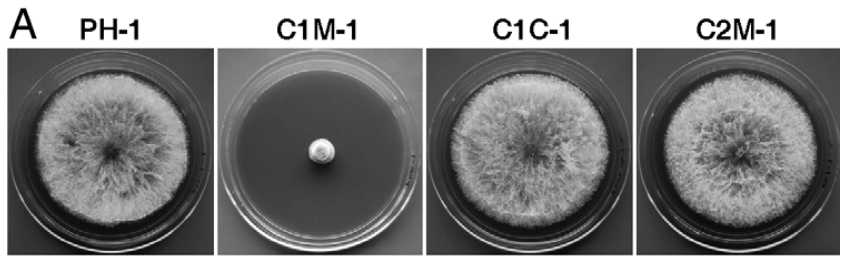

B

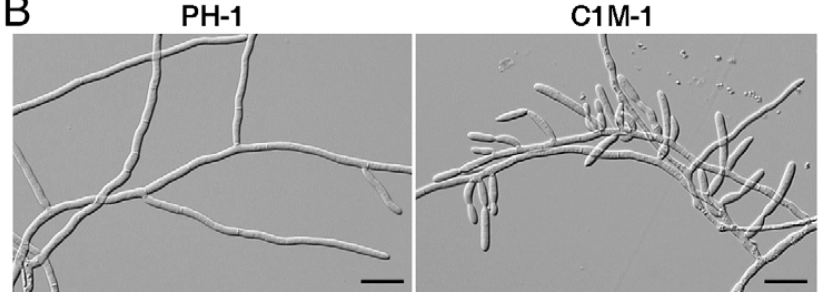

Fig. 1. The $c p k 1$ mutant was defective in colony morphology and hyphal growth. A, Colonies formed by the wild type PH-1, cpk1 mutant C1M-1, cpk 2 mutant C2M-1, and $c p k 1 / C P K 1$ complemented strain C1C-1 on potato dextrose agar plates. B, The formation of the conidiophores in the $\mathrm{PH}-1$ and $c p k 1$ mutant C1M-1 in CMC medium after incubation for $16 \mathrm{~h}$. Bar $=20 \mu \mathrm{m}$.

Table 1. Wild-type and mutant strains of Fusarium graminearum used in this study

\begin{tabular}{lll}
\hline Strains & \multicolumn{1}{c}{ Brief description } & Reference \\
\hline PH-1 & Wild-type & Cuomo et al. 2007 \\
C1M-1 & $c p k 1$ deletion mutant of PH-1 & This study \\
C1M-3 & $c p k 1$ deletion mutant of PH-1 & This study \\
C1M-6 & $c p k 1$ deletion mutant of PH-1 & This study \\
C1C-1 & $c p k 1 / C P K 1$ complemented transformant & This study \\
C2M-1 & $c p k 2$ deletion mutant of PH-1 & This study \\
C2M-7 & $c p k 2$ deletion mutant of PH-1 & This study \\
C2M-8 & $c p k 2$ deletion mutant of PH-1 & This study \\
C2M-14 & $c p k 2$ deletion mutant of PH-1 & This study \\
DM-1 & $c p k 1 c p k 2$ double mutant & This study \\
DM-8 & $c p k 1 c p k 2$ double mutant & This study \\
DM-9 & $c p k 1$ cpk2 double mutant & This study \\
FM-1 & $f a c 1$ deletion mutant of PH-1 & This study \\
FM-5 & $f a c l$ deletion mutant of PH-1 & This study \\
FM-7 & $f a c 1$ deletion mutant of PH-1 & This study \\
FC-1 & $f a c l / F A C 1$ complemented transformant & This study \\
\hline
\end{tabular}


Elevated expression levels of genes related to conidiation and secondary metabolism in the cpk1 mutant.

Several genes, including HTF1, MCM1, FgSTUA, and $\mathrm{FgCOS1}$, are known to be important for conidiation in $F$. graminearum (Lysøe et al. 2011; Zheng et al. 2012b; Zhou et al. 2011; Zhou et al. 2009). In RNA samples isolated from 12-h complete medium (CM) cultures, the expression levels of HTF1, MCM1, FgSTUA, and FgCOS1 were 57-, 12-, four-, and sevenfold, respectively, higher in the $c p k l$ mutant than in PH-1 (Fig. 2). These results confirmed that CPK1 plays a negative role in regulating conidiation under nutrient-rich conditions, which is consistent with the observation that the $c p k l$ mutant was depressed in conidiation.

Because conidiation is often related to secondary metabolism (Calvo et al. 2002), we also assayed the expression levels of the GIP1 laccase and GIP2 C6 zinc finger transcription factor genes that are involved in aurofusarin biosynthesis (Kim et al. 2005, 2006). In comparison with PH-1, the cpkl mutant had 11- and 232-fold increase in the expression of the GIP1 and GIP2 genes, respectively. Therefore, $C P K 1$ also negatively regulates the expression of $G I P 1, G I P 2$, and possibly other aurofusarin biosynthesis genes.

In addition, DON is an important secondary metabolite in $F$. graminearum; therefore, we assayed the expression levels of the TRI5, TRI6, and TRII2 genes in DON-producing cultures containing $5 \mathrm{mM}$ arginine by quantitative reverse-transcriptase polymerase chain reaction (qRT-PCR). In comparison with that of the wild type, the expression level of TRI5, TRI6, or TRI12 was reduced approximately fourfold in the $c p k 1$ mutant (Supplementary Fig. S2). In contrast, none of these three TRI genes had more than twofold reduction in the expression level in the cpk2 mutant. In the facl mutant, however, all of these three TRI genes were significantly reduced in expression and their transcripts were barely detectable.

\section{Altered heat shock responses in the cpk1 mutant.}

Because cAMP signaling is known to be involved in heat tolerance, we assayed conidium germination and hyphal growth at $35^{\circ} \mathrm{C}$. In the wild type, conidium germination was rarely observed, although conidium swelling and dead conidium compartments were observed after incubation in yeast extract peptone dextrose (YEPD) for $6 \mathrm{~h}$ or longer (Fig. 3A). Under the same conditions, conidium germination and germ tube growth were observed in the cpkl mutant (Fig. 3A). However, some conidium compartments and germ tubes became dead and empty by $24 \mathrm{~h}$. Germ tube tips also tended to swell in the $c p k 1$ mutant. When assayed for growth on potato dextrose agar (PDA) plates, the wild-type strain formed compact colonies with limited growth at $35^{\circ} \mathrm{C}$. The $c p k 1$ mutant had similar growth rate and colony morphology when cultured at 25 and $35^{\circ} \mathrm{C}$ (Fig. 3B). These results indicate that the cpkl mutant was more tolerant to heat stress than the wild type for conidium germination and hyphal growth.

To further verify increased tolerance of the $c p k l$ mutant to elevated temperatures, we assayed the expression of the FgHSP30 (Fg01158), FgHSP70 (Fg00838), and FgGSY2 (Fg06822) genes. The orthologs of these genes are known to be related to heat shock responses in other fungi (Choi and $\mathrm{Xu}$ 2010; Lindquist and Craig 1988). When cultured at $35^{\circ} \mathrm{C}$, the relative expression levels of FgHSP30, FgHSP70, and $F g G S Y 2$ were 70-, 11-, and sevenfold higher, respectively, in the cpkl mutant than in $\mathrm{PH}-1$. Therefore, deletion of $C P K 1$ resulted in elevated expression of heat stress response genes in F. graminearum.

\section{CPK1 is required}

for ascosporogenesis and ascospore releasing.

On carrot agar plates, both $c p k 1$ and $c p k 2$ mutants were able to produce normal perithecia, indicating that neither of these two PKA catalytic subunit genes is essential for homothallic sexual reproduction in $F$. graminearum. However, in comparison with the wild type and the $c p k 2$ mutant, the $c p k 1$ mutant produced fewer perithecia in self-crosses (Fig. 4A). In addition, only a few perithecia formed by the cpkl mutant produced diminished cirrhi that appeared as small, pale drops on top of perithecia at 2 to 3 weeks postinduction for sexual reproduction. Under the same conditions, the wild-type, cpk2 mutant,

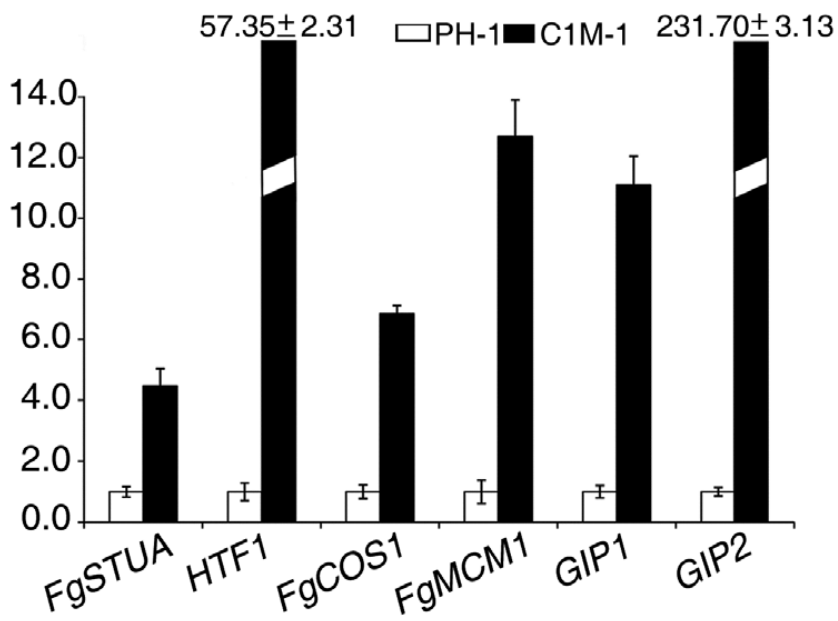

Fig. 2. Expression levels of genes related to conidiation and aurofusarin biosynthesis assayed by quantitative reverse-transcriptase polymerase chain reaction. RNA samples isolated from 12-h CM cultures of PH-1 and the cpkl mutant. Relative expression level of the FgSTUA (Fg10129), HTF1 (Fg16777), FgCOS1 (Fg16816), FgMCM1 (Fg08696), GIP1 (Fg02328), and GIP2 (Fg02320) genes in the wild type was arbitrarily set to 1 .

Table 2. Phenotypes of the $c p k 1, c p k 2$, and $f a c 1$ mutants in growth, conidiation, and plant infection

\begin{tabular}{|c|c|c|c|c|c|}
\hline \multirow[b]{2}{*}{ Strain } & \multirow[b]{2}{*}{ Growth rate $(\mathbf{m m} / \text { day })^{w, x}$} & \multirow[b]{2}{*}{ Conidiation $\left(\times 10^{5} \text { conidia/ml }\right)^{w}$} & \multirow[b]{2}{*}{ Disease inde $^{\mathrm{w}, \mathrm{y}}$} & \multicolumn{2}{|c|}{ DON $(\mathbf{p p m})^{\mathbf{w}, \mathbf{z}}$} \\
\hline & & & & Wheat & Rice \\
\hline PH-1 (wild type) & $11.6 \pm 0.3 \mathrm{a}$ & $12.5 \pm 1.8 \mathrm{~b}$ & $12.6 \pm 0.5 \mathrm{a}$ & $1,523.6 \pm 163.3 \mathrm{a}$ & $1,629.0 \pm 294.4 \mathrm{a}$ \\
\hline $\mathrm{C} 1 \mathrm{M}-1$ & $1.6 \pm 0.5 \mathrm{bc}$ & $7.0 \pm 0.5 \mathrm{c}$ & $1.0 \pm 0.0 \mathrm{~b}$ & $147.01 \pm 17.3 \mathrm{c}$ & $82.2 \pm 54.4 \mathrm{~b}$ \\
\hline C1C-1 & $11.4 \pm 0.9 \mathrm{a}$ & $12.5 \pm 1.3 \mathrm{~b}$ & $12.4 \pm 0.9 \mathrm{a}$ & $1,386.8 \pm 247.6 \mathrm{ab}$ & $1,396.8 \pm 293.0 \mathrm{a}$ \\
\hline $\mathrm{C} 2 \mathrm{M}-1$ & $11.3 \pm 0.3 \mathrm{a}$ & $17.7 \pm 2.4 \mathrm{a}$ & $13.0 \pm 0.8 \mathrm{a}$ & $1,179.2 \pm 102.4 \mathrm{~b}$ & $1,466.5 \pm 993.2 \mathrm{a}$ \\
\hline DM-1 & $0.8 \pm 0.4 \mathrm{c}$ & $2.0 \pm 0.5 \mathrm{~d}$ & $0.6 \pm 0.5 \mathrm{~b}$ & $0.0 \mathrm{c}$ & $0.0 \mathrm{~b}$ \\
\hline FM-1 & $2.0 \pm 0.5 \mathrm{~b}$ & $3.8 \pm 0.8 \mathrm{~d}$ & $0.5 \pm 0.6 \mathrm{~b}$ & $0.0 \mathrm{c}$ & $0.1 \pm 0.2 \mathrm{~b}$ \\
\hline
\end{tabular}

${ }^{\mathrm{w}}$ The same letter following values indicates there was no significant difference; different letters indicate statistically significant differences $(P=0.05)$.

${ }^{\mathrm{x}}$ Average daily extension of colony radium/conidiation and standard deviation (mean \pm standard deviation) were calculated from at least three independent measurements.

${ }^{y}$ Diseased spikelets per wheat head examined 14 dpi. Mean and standard deviation were calculated with results from three independent experiments.

${ }^{\mathrm{z}}$ Flowering wheat heads were inoculated and infected wheat kernels were harvested for deoxynivalenol (DON) assays 14 days postinoculation (dpi). Autoclaved rice grains were inoculated and cultured for 21 days, then assayed for DON. 
and complemented $c p k 1 / C P K 1$ strains produced extensive, yellowish cirrhi (Fig. 4A). These results indicate that the fertility of the cpkl mutant was reduced.

When examined under a compound microscope, perithecia produced by the cpkl mutant contained normal fascicles of asci, which had eight ascospores (Fig. 4B). However, many of the ascospores produced by the cpkl mutant strain $\mathrm{C} 1 \mathrm{M}-1$ were two-celled. Furthermore, mature ascospores of C1M-1 were often fragmented in the middle and each cell contained one or two nuclei visible by $4^{\prime}, 6$-diamindo-2-phenylindole

A

$6 \mathrm{~h}$

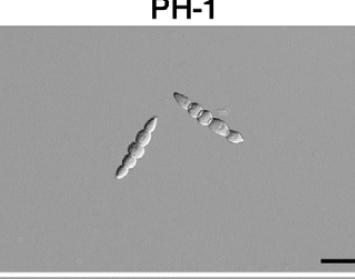

$12 \mathrm{~h}$

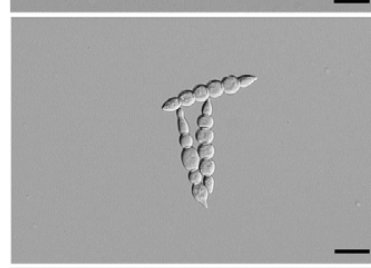

$24 \mathrm{~h}$

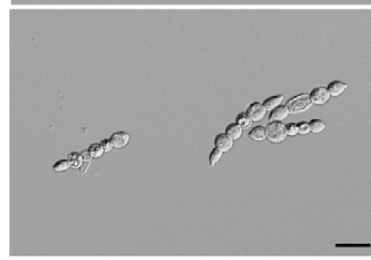

B

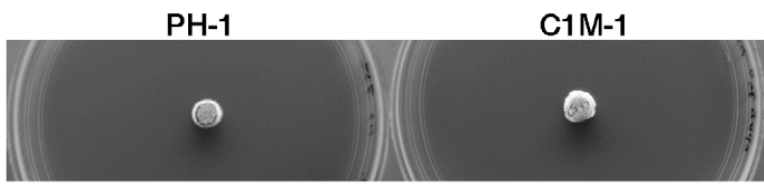

C

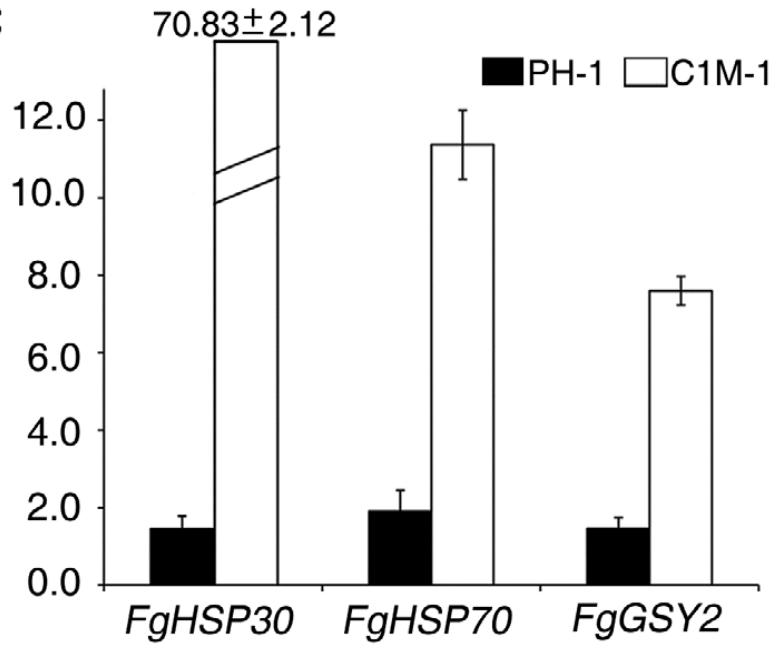

Fig. 3. The $c p k 1$ mutant has increased tolerance to elevated temperatures A, Conidia of PH-1 and the cpkl mutant were incubated in YEPD medium at $35^{\circ} \mathrm{C}$. Germination and germ tube growth were examined after incubation for 6,12 , and $24 \mathrm{~h}$. Bar $=20 \mu \mathrm{m}$. B, Colonies formed by PH-1 and the cpk1 mutant $\mathrm{C} 1 \mathrm{M}-1$ on potato dextrose agar plates at $35^{\circ} \mathrm{C}$. C, Quantitative reverse-transcriptase polymerase chain reaction assays for the relative expression of FgHSP30 (Fg01158), FgHSP70 (Fg00838), and FgGSY2 (Fg06822). RNA samples were isolated from 12-h germlings harvested from CM cultures of PH-1 and C1M-1 that were incubated for $11 \mathrm{~h}$ at $25^{\circ} \mathrm{C}$ before switching to $35^{\circ} \mathrm{C}$ for $1 \mathrm{~h}$. The expression level of each gene at $25^{\circ} \mathrm{C}$ for $12 \mathrm{~h}$ was arbitrarily set to 1 . staining (Fig. 4C). In the $c p k 1 / C P K 1$ transformant, ascospore morphology was normal. Therefore, although it is dispensable for ascus development and initial ascospore formation, $C P K 1$ is important for ascospore maturation and release in $\mathrm{F}$. graminearum.

\section{CPK1 is important for virulence.}

In infection assays with flowering wheat heads, the wild type could cause typical head blight symptoms in the inoculated kernels and spread to other spikelets. On wheat heads inoculated with the cpkl mutant, only the inoculated floret developed scab symptoms 14 days postinoculation (dpi). It failed to spread to nearby spikelets (Fig. 5A). As a matter of fact, most of the time the $c p k 1$ mutant failed to spread from the inoculated kernel to the other two florets on the same spikelet. In contrast, the complemented strain $\mathrm{C} 1 \mathrm{C}-1$ and the $c p k 2$ mutant were as virulent as the wild type (Fig. 5A). The average disease index of the cpkl and cpk2 mutants and PH-1 was 1.0, 13.0, and 12.6, respectively (Table 2 ).

Because DON is an important virulence factor, we assayed DON production in the $c p k 1$ and $c p k 2$ mutants. Whereas the $c p k 2$ mutant was normal, the $c p k 1$ mutant was significantly reduced in DON production in infested wheat kernels (Table 2). To confirm this observation, we assayed DON production in rice grain cultures as described (Bluhm et al. 2007; Seo et al. 1996). Similar to results with infested wheat grains, the $c p k l$ mutant was significantly reduced in DON production in rice grain cultures but the cpkl cpk2 double mutant and the facl mutant were blocked in DON biosynthesis (Table 2). DON production in these mutants is consistent with reduced expression of TRI genes, indicating that $C P K I$ and FAC1 play an important role in the regulation of $T R I$ gene expression and DON biosynthesis.
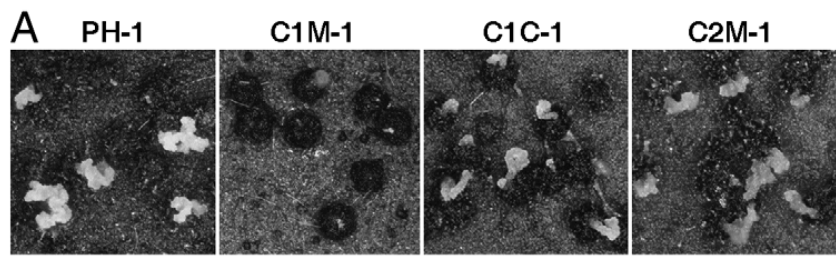

B
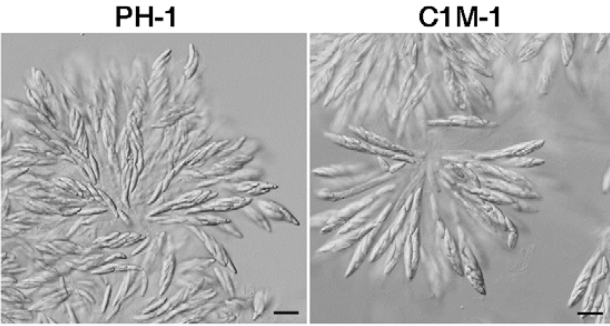

$\mathrm{C}$
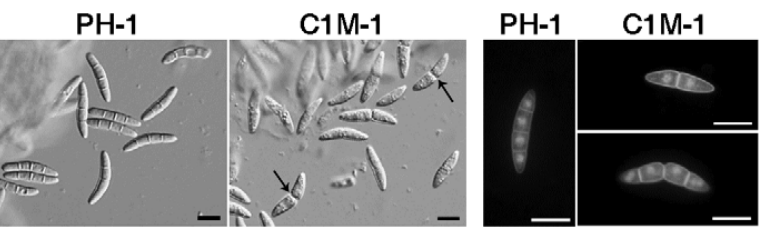

Fig. 4. Ascospore maturation and release require $C p k 1$ but not $C p k 2$. A, Mating cultures of $\mathrm{PH}-1, c p k 1$ or $c p k 2$ mutant, and complemented $c p k 1 / C P K 1$ transformant were examined 2 weeks postinduction for sexual reproduction. The $c p k 1$ mutant produced fewer perithecia and rarely formed small, pale cirrhi. B, Asci and ascospores from perithecia formed by the PH-1 and the cpkl mutant. Bar $=20 \mu \mathrm{m}$. C, Ascospores of PH-1 and the cpkl mutant C1M-1 were stained with 4',6-diamindo-2-phenylindole and Calcofluor white. Ascospores produced by the cpk1 mutant were often two-celled and fragmented in the middle. Bar $=10 \mu \mathrm{m}$. 
$C P K 1$ is important for plant colonization.

To further determine the function of $C P K 1$ in plant infection, we examined the defects of the $c p k l$ mutant in different infection processes. At $24 \mathrm{~h}$ postinoculation (hpi), direct penetration of wheat lemma epidermis by short, penetration branches (Wanjiru et al. 2002) was observed in the wild-type strain by scanning electron microscopy (SEM). The cpkl mutant was significantly reduced in the production of penetration branches and most of the germ tubes failed to penetrate underlying plant cells (Fig. 5B). By 48 hpi, extensive hyphal growth was observed in lemma tissues of wheat heads inoculated with $\mathrm{PH}-1$. In contrast, fungal growth was restricted to the inner epidermal tissues in samples inoculated with the $c p k 1$ mutant (Fig. 5C). These results indicate that the cpkl mutant was reduced in the formation of penetration hyphae and delayed in the initial infection processes.

In addition to spreading to nearby spikelet is via the rachis, we also examined for fungal growth in the rachis of inoculated wheat heads. By 120 hpi, extensive fungal growth was observed in the vascular tissues of the rachis in wheat heads inoculated with PH-1. In wheat heads infected with the cpkl mutant, we failed to observe fungal hyphae in the rachis below or above the inoculated spikelet (Fig. 5D). Therefore, $C P K 1$ must be essential for spreading from inoculated florets to the rachis and colonization of vascular tissues in F. graminearum.

\section{Deletion of CPK1 or CPK2 had only minor effects on the expression of the other $C P K$ gene.}

To determine whether deletion of $C P K 1$ affects the expression of $C P K 2$ or vice versa, RNA samples were isolated from germlings or vegetative hyphae of $3,6,12,18,24$, and $48 \mathrm{~h}$ in $\mathrm{CM}$ cultures of $\mathrm{PH}-1$ and the $c p k 1$ and $c p k 2$ mutants. The expression levels of $C P K 1$ and $C P K 2$ were assayed by qRT-PCR. In the wild type, the $C P K 1$ gene had the lowest expression level at $3 \mathrm{~h}$ and its expression increased over time (Fig. 6A). In comparison with 3-h germlings, $C P K 1$ expression increased more than twofold from 6 to $12 \mathrm{~h}$ (Fig. 6A). The $C P K 2$ gene had an expression profile similar to $C P K 1$ in the wild type but its expression level was lower than that of $C P K 1$ in germ tubes and hyphae, particularly at 6,12 , and $18 \mathrm{~h}$ (Fig. 6B).

In comparison with the wild type, the expression level of $C P K 1$ was increased in the $c p k 2$ mutant but the increase was less than twofold in all the time points assayed by qRT-PCR (Fig. 6C). In the cpkl mutant, the expression level of $C P K 2$ was increased slightly at 3 and $6 \mathrm{~h}$ (Fig. 6C) but reduced in other time points assayed. Therefore, deletion of $C P K 1$ or $C P K 2$ had only a minor effect (less than twofold) on the expression of the other PKA catalytic subunit gene in F. graminearum.

\section{The cpk1 cpk2 double mutant has severe defects in growth and conidiogenesis.}

Based on the phenotypes of the cpkl and cpk2 mutants, $C P K 1$ must encode the major catalytic subunit of PKA and $C P K 2$ plays only a minor role in $F$. graminearum. To further examine their functional relationship, we generated the $c p k 1$ $c p k 2$ double mutant by deletion of $C P K 1$ in the $c p k 2$ mutant C2M-1 (Supplementary Fig. S3). The resulting double mutants (Table 1) were more significantly reduced in vegetative growth than the cpkl mutant (Fig. 7A; Table 2). Whereas the cpkl mutant still produced normal conidia and appeared to be de-repressed in conidiation in nutrient-rich conditions, the cpk1 cpk2 double mutant rarely produced conidia (Table 2) that had abnormal morphology (Fig. 7B). In general, conidia produced by the cpk1 cpk2 double mutant were smaller or shorter than the wild-type conidia. Many conidia produced by the double mutant had only one or two compartments (Fig. 7B).
The cpk1 cpk2 double mutant

is nonpathogenic and sterile in self-crosses.

In infection assays with flowering wheat heads, the cpkl cpk2 mutant was nonpathogenic and failed to colonize the inoculated florets (Fig. 7C). The double mutant did not produce or produced barely detectable amount of DON (Table 2). The defects in growth and DON production may contribute to the loss of pathogenicity in the cpkl cpk2 mutant.

Because the $c p k 1$ mutant was defective in ascosporogenesis, we also assayed the defect of the $c p k 1 c p k 2$ mutant in sexual
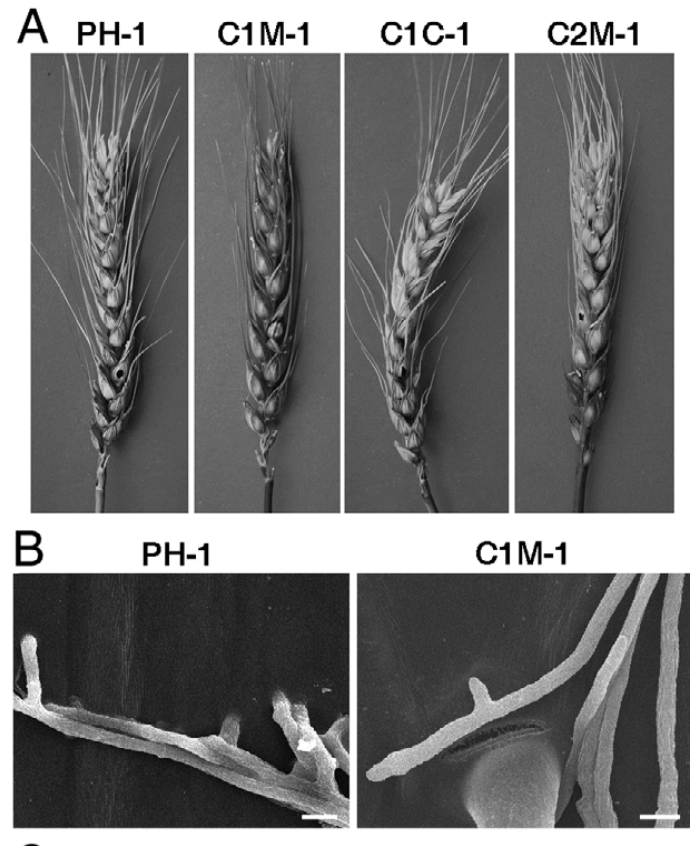

C
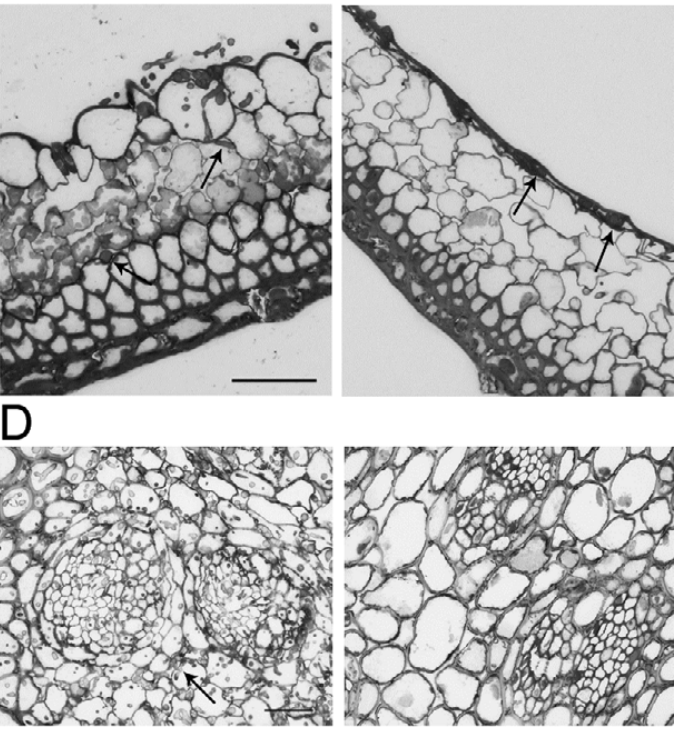

Fig. 5. Defects of the $c p k 1$ mutant in plant infection. A, Flowering wheat heads were drop-inoculated with conidia from the wild type (PH-1), cpk1 mutant (C1M-1), cpk2 mutant (C2M-1), and complemented cpk1/CPK1 transformant (C1C-1). Black dots mark the inoculated spikelets. Photographs were taken 14 days postinoculation. B, Lemma from the spikelets inoculated with PH-1 and C1M-1 were examined by SEM $24 \mathrm{~h}$ postinoculation (hpi). Bar $=5 \mu \mathrm{m}$. The $c p k 1$ mutant was defective in the production of penetration branches. C, Colonization of lemma tissues by PH-1 and the cpkl mutant 48 hpi. Fungal hyphae were marked with arrows. Bar $=50 \mu \mathrm{m}$. D, Rachises next to the inoculated spikelets were examined $120 \mathrm{hpi}$. Extensive hyphal growth in plant tissues (marked with arrows) were observed in samples inoculated with $\mathrm{PH}-1$ but the $c p k 1$ mutant. $\mathrm{Bar}=50 \mu \mathrm{m}$. 
reproduction. To our surprise, the $c p k 1 c p k 2$ mutant was sterile in self-crosses. Although neither $C P K 1$ nor $C P K 2$ is essential for perithecium formation, the $c p k 1$ cpk2 mutant DM-1 failed to produce perithecia or protoperithecia on carrot agar cultures (Fig. 7D). The other two cpkl cpk2 double mutants (Table 1) had phenotypes similar to that of mutant DM-1. These results indicate that the double mutant displayed more severe defects in pathogenesis and sexual reproduction than the $c p k l$ and cpk2 mutants. Therefore, $C P K 1$ and $C P K 2$ must have overlapping functions in $F$. graminearum during plant infection and differentiation.

\section{The fac1 mutant differs}

from the cpk1 cpk2 mutant in conidiation.

In addition to functionally characterizing the $C P K 1$ and $C P K 2$ genes, we also generated the knockout mutant of the FAC1 (FGSG_01234) adenylate cyclase gene by the gene replacement approach. The facl deletion mutant formed small, compact colonies with limited aerial hyphal growth (Fig. 8A).
In the wheat head and corn silk infection assays, the facl mutant was nonpathogenic (Fig. 8B) and did not produce detectable amounts of DON (Table 2). These results indicate that the facl mutant and cpkl cpk2 double mutant had similar defects in growth and pathogenesis. However, unlike the double mutant, the facl mutant FM-1 produced morphologically normal conidia (Fig. 8C), although conidiation was significantly reduced (Table 2). The other two facl mutants, FM-5 and FM-7 (Table 1), had defects similar to those of mutant FM-1.

\section{DISCUSSION}

The cAMP-PKA signaling pathway is involved in growth, development, nutrient sensing, mating, stress response, secondary metabolism, and pathogenicity in various fungi (Banno et al. 2005; Cervantes-Chavez et al. 2009; D'Souza and Heitman 2001; Lee et al. 2003; Lengeler et al. 2000; Liebmann et al. 2004). In many filamentous ascomycetes, including $A$. nidulans, $N$. crassa, and $M$. oryzae, there are two catalytic subunit genes
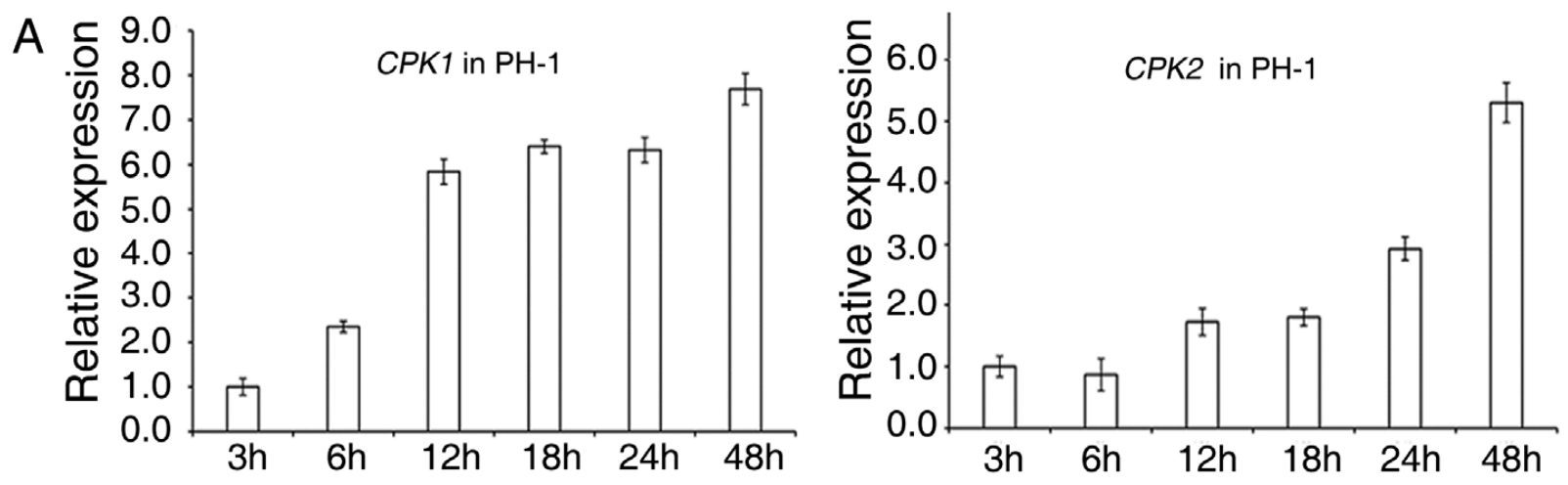

B
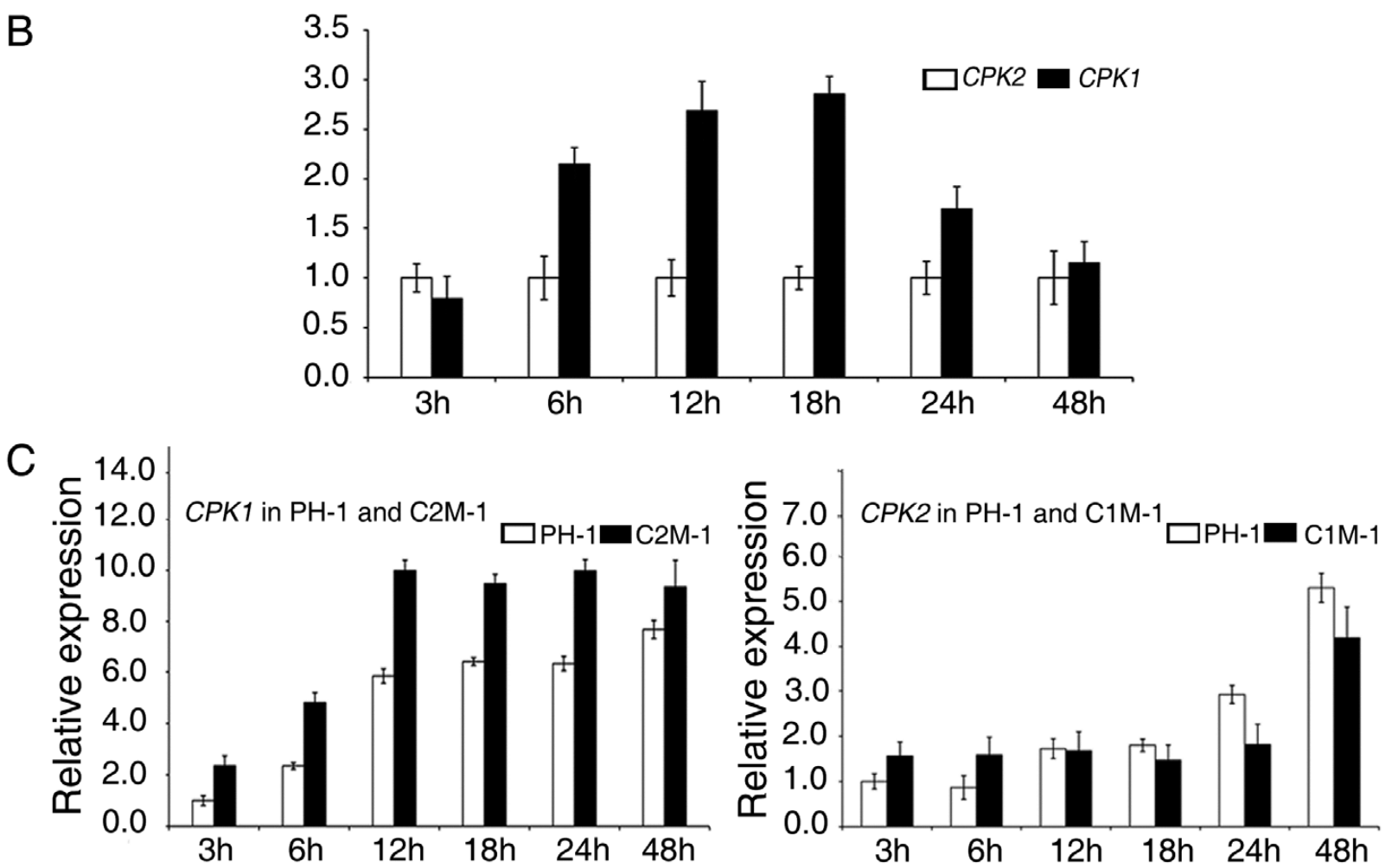

Fig. 6. Expression levels of the $C P K 1$ and $C P K 2$ genes assayed by quantitative reverse-transcriptase polymerase chain reaction. A, RNA samples were isolated from conidia of PH-1 cultured in YEPD medium for $3,6,12,18,24$, and $48 \mathrm{~h}$ at $25^{\circ} \mathrm{C}$. Relative expression levels of $C P K 1$ and $C P K 2$ were calculated with $G z U B H$ gene as the internal control using the $2^{-\triangle \Delta C T}$ method (Kim and Yun 2011). The expression level of $C P K 1$ or $C P K 2$ at $3 \mathrm{~h}$ was arbitrarily set to 1 . $\mathbf{B}$, Relative expression levels of $C P K 1$ in comparison with $C P K 2$. The expression level of $C P K 2$ was set to 1 for all the samples. C, Relative expression levels of $C P K 1$ and $C P K 2$ in the $c p k 2$ and $c p k 1$ mutants, respectively. Their expression level in PH-1 was arbitrarily set to 1. 
and, usually, one of them is responsible for most of the PKA activity. The other one plays only a minor role or has no detectable functions (Lee et al. 2003; Ni et al. 2005; Schumacher et al. 2008). In $F$. graminearum, $C P K 1$ is orthologous to the major PKA catalytic subunit genes that have been characterized in other fungi and it plays a critical role in vegetative growth, asexual reproduction, sexual reproduction, and plant infection. In contrast, deletion of the minor PKA subunit gene $C P K 2$ had no detectable effects. It appears that the $C P K 1$ and $C P K 2$ genes do not regulate each other transcriptionally because deletion of one of them had no significant effect (more than twofold) on the expression of the other.

Interestingly, the cpkl mutant was reduced over $90 \%$ in growth rate but the conidiation level was only reduced by approximately $44 \%$. Close examination indicated that the $c p k 1$ mutant formed abundant phialides at $16 \mathrm{~h}$ in CMC cultures and began to sporulate earlier than the wild type (Fig. 1). Suppression of conidiation in liquid cultures in the wild type is due to the presence of sufficient growth nutrients (Adams et al. 1998). Nutrients in CMC after $16 \mathrm{~h}$ of growth of the cpkl mutant were likely abundant, particularly because it had a reduced growth rate; therefore, conidiation should be suppressed, at least similarly to that of the wild type. Therefore, earlier conidiation observed in the cpkl mutant may be due to a defect in nutrient sensing, which leads to a de-repression of asexual reproduction. In poor nutrient medium, such as mung bean liquid medium, the cpkl mutant and $\mathrm{PH}-1$ began to produce phialides and conidia at the same time (Supplementary Fig. S4). Conidiation also is de-repressed in the $c r-1$ mutant of $N$. crassa (Banno et al. 2005) and the pkaA gene is known to negatively regulate asexual reproduction in $A$. nidulans $(\mathrm{Ni}$ et al. 2005). In F. graminearum, several transcription factor genes are known to play regulatory roles in conidiation. The expression of the HTF1, MCM1, FgSTUA, and FgCOS1 genes was upregulated in the cpkl mutant, suggesting that deletion of $C P K 1$ may result in de-repression of conidiation in the presence of abundant nutrients. Nevertheless, as in many other fungal pathogens (Choi and Xu 2010; Tzima et al. 2010), deletion of the CPKA ortholog resulted in a reduction in conidiation in $F$. graminearum in 5-day-old CMC cultures. In addition, the cpk1 cpk2 double mutant rarely produced conidia. Therefore, normal or efficient production of conidiation still requires proper regulation by the cAMP-PKA pathway.
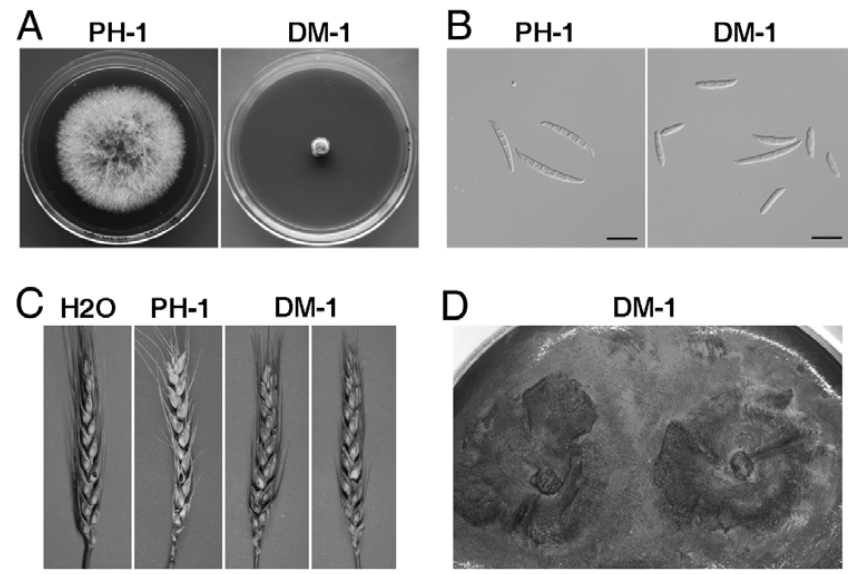

Fig. 7. Defects of the $c p k 1 c p k 2$ double mutant in growth, pathogenesis, sexual, and asexual reproduction. A, Colonies formed by $\mathrm{PH}-1$ and the cpk1 cpk2 double mutant DM-1 on 3-day-old potato dextrose agar cultures. B, Conidia of $\mathrm{PH}-1$ and the $c p k 1$ cpk2 mutant in $\mathrm{CMC}$ cultures after 5 days. Bar $=20 \mu \mathrm{m}$. C, Flowering wheat heads inoculated with PH-1 and the $c p k 1$ cpk2 mutant. Inoculation with $\mathrm{H}_{2} \mathrm{O}$ was used as the negative control. D, Two-week-old carrot agar cultures of the $c p k 1$ cpk2 mutant.
Similar to its role in suppressing conidiation under nutrientrich conditions, $C P K 1$ may also negatively regulate responses to heat stresses in $F$. graminearum. In comparison with the wild type, the cpkl mutant had increased heat tolerance and upregulated expression of heat shock protein genes $\mathrm{FgHSP} 30$, FgHSP70, and FgGSY2 (Fig. 3C). In F. verticillioides and $N$. crassa, mutants defects in cAMP signaling also display increased tolerance to elevated temperatures (Supplementary Table S1) (Banno et al. 2005; Choi and Xu 2010).

As the primary inoculum, ascospores play a critical role in the disease cycle of $F$. graminearum. The cpkl mutant produced morphologically normal perithecia but it was reduced in perithecium formation and defective in cirrhi development in self-crosses. Most of the mature ascospores produced by the cpkl mutant were often two-celled and fragmented in the middle, suggesting that the cAMP-PKA pathway may be involved in ascosporogenesis in $F$. graminearum. In several filamentous fungi, orthologs of Cpk1 are known to play an important role in sexual development (D'Souza and Heitman 2001; Lengeler et al. 2000). However, to our knowledge, the involvement of Cpk1 in ascosporogenesis has not been reported. Fragmented ascospores suggest that the constriction and septum separating two daughter cells after the first mitosis in developing ascospores became complete in the cpkl mutant. Therefore, CPK1 may be involved in regulating late stages of ascospore formation, particularly ascospore maturation. In $F$. graminearum, deletion of $M I D 1$, a mechanosensitive calcium ion channel gene, resulted in a reduction in forcible ascospore discharge and the production of abnormal, two-celled ascospores with constricted and fragile septa (Cavinder et al. 2011). In filamentous fungi, calcium signaling plays a major role in regulating
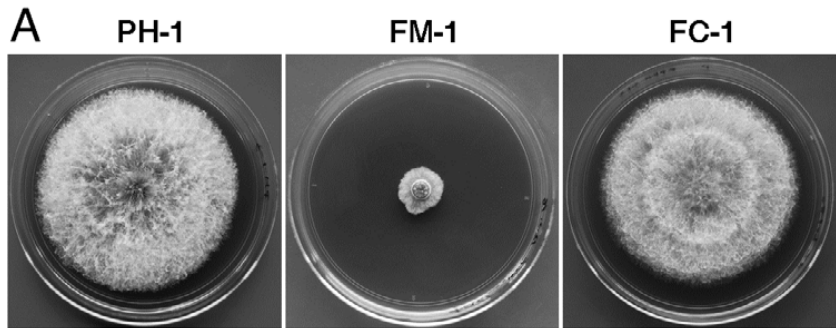

B
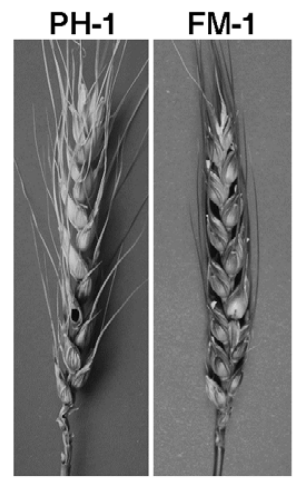

PH-1 FM-1
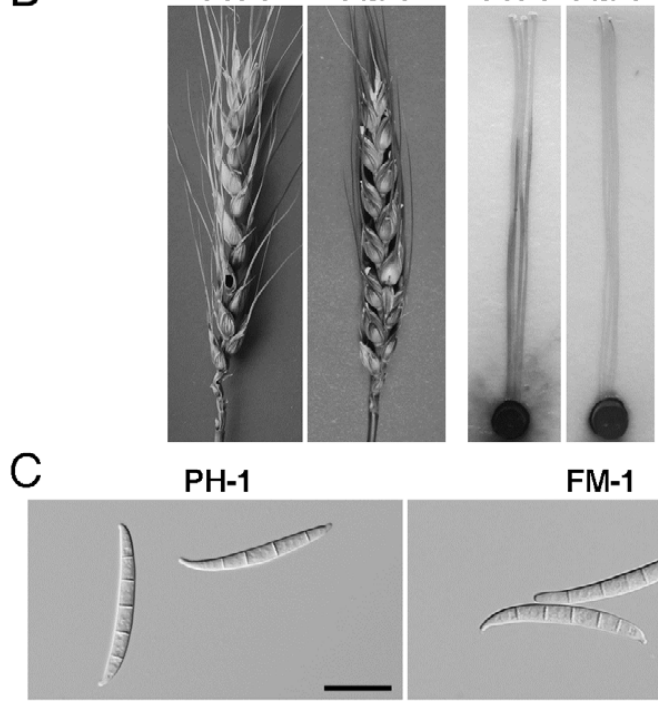

C

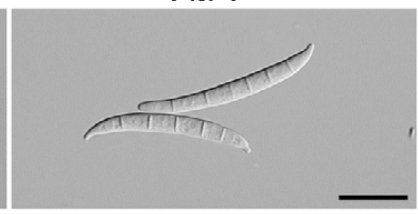

Fig. 8. Defects of the $f a c 1$ mutant in growth and virulence. A, Three-day old potato dextrose agar cultures of the wild type (PH-1), facl mutant (FM-1), and facl/FACl complemented transformant (FC-1). B, Wheat heads and corn silks inoculated with PH-1and FM-1. C, Conidia of PH-1 and the $f a c 1$ mutant in $\mathrm{CMC}$ cultures after 5 days. Bar $=20 \mu \mathrm{m}$. 
hyphal tip growth, branching, dimorphism, and the cell cycle (Bencina et al. 2005; Cavinder and Trail 2012). PKA-dependent phosphorylation regulates $\mathrm{Ca}^{2+}$-channel activities in $A$. niger (Bencina et al. 2005). The defects of ascospores development and release in the cpkl mutant were similar to those of the midl mutant, indicating that the cAMP-PKA pathway may cross-talk with $\mathrm{Ca}^{2+}$ signaling in $F$. graminearum.

As in many other plant-pathogenic fungi (Fuller and Rhodes 2012; Lee et al. 2003; Lengeler et al. 2000), CPK1 and FAC1 were found to be important for plant infection in $F$. graminearum. SEM examination revealed that deletion of the CPKI genes resulted in a significant reduction in the formation of penetration hyphae (Fig. 5B). In M. oryzae and other fungal pathogens, cAMP signaling is involved in surface recognition and plant-pathogen interaction (Lee et al. 2003; Ni et al. 2005; Schumacher et al. 2008). In $F$. graminearum, the cAMP-PKA pathway may also regulate the recognition and development of plant infection structures. In addition, the cpkl and facl mutants were reduced or blocked in the production of DON (Table 2), which is one of the best characterized virulence factors in F. graminearum (Proctor et al. 1995). Defects in DON production may be directly responsible for the phenotypes of these mutants in spreading in flowering wheat heads and colonization of vascular tissues in the rachis. However, the cpkl and facl mutants were reduced in growth rate, which may also contribute to their defects in plant infection.

In $M$. oryzae, deletion of the $C P K A$ or $M A C l$ gene does not affect growth rate but results in significant reduction in aerial hyphae (Adachi and Hamer 1998; Choi and Dean 1997; Mitchell and Dean 1995). However, in other filamentous fungi such as $F$. verticillioides, $N$. crassa, A. nidulans, and $F$. oxysporum, the adenylate cyclase mutant or PKA catalytic subunit mutant had significantly reduced growth rate (Choi and $\mathrm{Xu}$ 2010; Kim et al. 2011). These results indicated that the role of cAMP signaling in vegetative growth is different among diverse fungal species. In $F$. verticillioides, the facl mutant grew more slowly than the cpkl mutant. However, in this study, we found that the cpkl cpk2 double mutant had more severe defects in conidiation and virulence than the facl mutant. Due to the conserved nature of the cAMP-PKA pathway, it is unlikely that the PKA catalytic subunit genes are activated by other mechanisms. The catalytic submits of PKA in $F$. graminearum may have residential activities without the binding of cAMP to the regulatory subunit of PKA. PKA may maintain a basal level activity even in the absence of $F A C l$ and cAMP in $F$. graminearum (Ni et al. 2005).

Although deletion of both catalytic subunits of PKA (Adr1 and Uka1) was not lethal in the basidiomycete pathogen $U$. maydis (Durrenberger et al. 1998), the tpk1 tpk2 tpk3 triple mutant was not viable in Saccharomyces cerevisiae (Toda et al. 1987). In $A$. nidulans, the overexpression of $p k a B$ can rescue the growth and spore germination defects caused by $\Delta p k a A$, and deletion of both $p k a B$ and $p k a A$ is lethal, indicating that PkaA and $\mathrm{PkaB}$ have overlapping functions (Ni et al. 2005). It has been generally assumed that the cpkl cpk2 double mutant was nonviable in $M$. oryzae (Choi and Xu 2010). In this study, we found that the cpk1 cpk2 mutant was viable, which is the first report of mutants with both PKA catalytic subunit genes deleted in filamentous ascomycetes. In comparison with the cpkl mutant, the double mutant had more severe defects in growth, sexual reproduction, and conidiation. It rarely produced conidia (Fig. 7B) and was nonpathogenic and sterile in self-crosses. In U. maydis, Adr1 is the major catalytic subunit of PKA but the adrl ukal double mutant also had more severe defects than the adrl (Durrenberger et al. 1998). In F. graminearum and possibly other filamentous ascomycetes, the two catalytic subunits of PKA likely have overlapping functions in regulating hyphal growth, sexual and asexual reproduction, and plant infection. Therefore, it will be important to characterize substrate specificities of $C P K 1$ and $C P K 2$ and their contributions to PKA activities in different developmental and infection processes.

\section{MATERIALS AND METHODS}

\section{Strains and culture conditions.}

The wild-type strain PH-1 (Cuomo et al. 2007) and mutants of $F$. graminearum generated in this study (Table 1) were routinely cultured at $25^{\circ} \mathrm{C}$ on PDA agar plates. Growth rate on PDA plates and conidiation in CMC medium were assayed as described previously (Ding et al. 2009; Zhou et al. 2010). For DNA and RNA extraction, vegetative hyphae or germlings were harvested from liquid YEPD (1\% yeast extract, $2 \%$ peptone, and $2 \%$ glucose). Protoplast preparation and fungal transformation were performed as described (Hou et al. 2002; Proctor et al. 1995). For transformation, hygromycin B (CalBiochem, La Jolla, CA, U.S.A.) and geneticin (Sigma-Aldrich, St. Louis) were added to the final concentration at 300 and 350 $\mu \mathrm{g} / \mathrm{ml}$, respectively.

\section{Generation of the cpk1 cpk2 and fac1 mutants.}

The cpk 1 and $c p k 2$ mutants were generated in a previous study by the split-marker approach (Wang et al. 2011). The same method was used to generate the $f a c l$ deletion mutant by transforming the overlapping PCR products into the protoplasts of PH-1. For generating the cpkl cpk2 double mutants, the $C P K 1$ gene-replacement construct generated with the neomycin resistance gene $\left(N E O^{R}\right)$ was transformed into the $c p k 2$ mutant C2M-1. The putative knockout mutants were identified by PCR and further confirmed by Southern blot hybridizations.

For complementation assays, the entire CPK1 gene was amplified with primers CM-CPK1/F and CM-CPK1/R, digested with $S a c \mathrm{I}$ and $E c o$ RI, and cloned into the $N E O^{R}$ vector pHZ100 (Bluhm et al. 2007). The resulting construct, pCPK1, was transformed into protoplasts of the cpkl mutant C1M-1. The same method was used to generate the fac1/FAC1 complemented transformants.

\section{Sexual reproduction assays.}

To induce sexual reproduction, aerial hyphae of 7-day-old carrot agar cultures were pressed down with sterile $0.1 \%$ Tween 20 and incubated at $25^{\circ} \mathrm{C}$, as described (Wang et al. 2011; Zheng et al. 2013). Perithecium formation, cirrhi production, and ascospores were examined 2 to 3 weeks after induction. Each experiment was repeated independently three times.

\section{Plant infection and DON production assays.}

Conidia of PH-1 and mutant strains were harvested from 5day-old CMC cultures and resuspended to $10^{6}$ spores $/ \mathrm{ml}$, as described (Hou et al. 2002). Flowering wheat heads of 'Norm' were inoculated with $10 \mu \mathrm{l}$ of conidium suspensions at the fifth spikelet from the base of the inflorescence, as described (Ding et al. 2009; Gale et al. 2007). Spikelets with typical head blight symptoms were examined 14 dpi and infected wheat kernels were harvested for DON assays, as described (Bluhm et al. 2007). For infection assays with mature corn plants of 'Pioneer 2375', sterile toothpicks were soaked in conidium suspensions before being pushed into the stalks as described (Choi and Xu 2010). Infection assays with corn silks were conducted as described (Seong et al. 2005). DON production in rice grain cultures was assayed as described (Bluhm et al. 2007; Seo et al. 1996). All the infection and DON production assays were repeated at least three times. 


\section{Microscopic observations.}

Glumes and lemmas were collected from inoculated spikelets and fixed with $4 \%$ (vol/vol) glutaraldehyde in $0.1 \mathrm{M}$ phosphate buffer ( $\mathrm{pH} 6.8)$ overnight at $4^{\circ} \mathrm{C}$. After dehydration in a series of acetone $(30,50,70,80,90$, and $100 \%$ [vol/vol] $)$, the samples were mounted on stubs, sputter coated with gold-palladium, and examined with a JEOL 6360 scanning electron microscope (Jeol Ltd., Tokyo). For light microscope examination, infected wheat lemma and rachis tissues were fixed, dehydrated, and embedded in Spurr resin, as described (Kang et al. 2008; Zheng et al. 2012a). Thick sections $(1 \mu \mathrm{m})$ were stained with $0.5 \%$ (wt/vol) toluidine blue and examined with an Olympus BX-53 microscope (Olympus, Tokyo). At least three independent biological replicates were examined for the wild-type and mutant strains.

\section{qRT-PCR analysis.}

Vegetative hyphae or germlings were isolated from CM cultures of $\mathrm{PH}-1$ and the $c p k 1$ or $c p k 2$ mutant $\left(10^{6}\right.$ conidia per $100 \mathrm{ml}$ ) after shaking at $175 \mathrm{rpm}$ for $3,6,12,18,24$, and $48 \mathrm{~h}$ at $25^{\circ} \mathrm{C}$. DON production and TRI gene expression in the presence of $5 \mathrm{mM}$ arginine were assayed as described (Gardiner et al. 2009). RNA was isolated with the TRIzol reagent (Invitrogen, Carlsbad, CA, U.S.A.). First-strand cDNA was synthesized with the Fermentas First cDNA synthesis kit (Hanover, MD, U.S.A.) following the instructions provided by the manufacturer. The $F$. graminearum ubiquitin $C$-terminal hydrolase $(G z U B H)$ gene was amplified with primers GzUBHQF and GzUBHQR (Kim and Yun 2011). Relative changes in the expression level of each gene were calculated by the $2^{-\Delta \Delta \mathrm{Ct}}$ method (Livak and Schmittgen 2001) with GzUBH as the endogenous reference. For each gene, qRT-PCR data from three biological replicates were used to calculate the mean and standard deviation with two technical repeats each.

\section{ACKNOWLEDGMENTS}

We thank H. Liu and Q. Jin for fruitful discussions and D. Zheng for assistance with qRT-PCR analysis. This work was supported by the National Major Project of Breeding for New Transgenic Organisms (2012ZX08009003) and the National Basic Research Program of China (2012CB114002; 2013CB127703).

\section{LITERATURE CITED}

Adachi, K., and Hamer, J. E. 1998. Divergent cAMP signaling pathways regulate growth and pathogenesis in the rice blast fungus Magnaporthe grisea. Plant Cell 10:1361-1373.

Adams, T. H., Wieser, J. K., and Yu, J. H. 1998. Asexual sporulation in Aspergillus nidulans. Microbiol. Mol. Biol. Rev. 62:35-54.

Alspaugh, J. A., Pukkila-Worley, R., Harashima, T., Cavallo, L. M., Funnell, D., Cox, G. M., Perfect, J. R., Kronstad, J. W., and Heitman, J. 2002. Adenylyl cyclase functions downstream of the $\mathrm{G}$ alpha protein Gpa1 and controls mating and pathogenicity of Cryptococcus neoformans. Eukaryot. Cell 1:75-84.

Bai, G. H., and Shaner, G. 2004. Management and resistance in wheat and barley to Fusarium head blight. Annu. Rev. Phytopathol. 42:135-161.

Banno, S., Ochiai, N., Noguchi, R., Kimura, M., Yamaguchi, I., Kanzaki, S., Murayama, T., and Fujimura, M. 2005. A catalytic subunit of cyclic AMP-dependent protein kinase, PKAC-1, regulates asexual differentiation in Neurospora crassa. Genes Genet. Syst. 80:25-34.

Bencina, M., Legisa, M., and Read, N. D. 2005. Cross-talk between cAMP and calcium signalling in Aspergillus niger. Mol. Microbiol. 56:268281 .

Bluhm, B. H., Zhao, X., Flaherty, J. E., Xu, J. R., and Dunkle, L. D. 2007. RAS2 regulates growth and pathogenesis in Fusarium graminearum. Mol. Plant-Microbe Interact. 20:627-636.

Brakhage, A. A., and Liebmann, B. 2005. Aspergillus fumigatus conidial pigment and cAMP signal transduction: Significance for virulence. Med. Mycol. 43:S75-S82.

Calvo, A. M., Wilson, R. A., Bok, J. W., and Keller, N. P. 2002. Relationship between secondary metabolism and fungal development. Micro- biol. Mol. Biol. Rev. 66:447-459.

Cavinder, B., and Trail, F. 2012. Role of Fig1, a component of the lowaffinity calcium uptake system, in growth and sexual development of filamentous fungi. Eukaryot. Cell 11:978-988.

Cavinder, B., Hamam, A., Lew, R. R., and Trail, F. 2011. Mid1, a mechanosensitive calcium ion channel, affects growth, development, and ascospore discharge in the filamentous fungus Gibberella zeae. Eukaryot. Cell 10:832-841.

Cervantes-Chavez, J. A., Kronberg, F., Passeron, S., and Ruiz-Herrera, J. 2009. Regulatory role of the PKA pathway in dimorphism and mating in Yarrowia lipolytica. Fungal Genet. Biol. 46:390-399.

Choi, W. B., and Dean, R. A. 1997. The adenylate cyclase gene MAC1 of Magnaporthe grisea controls appressorium formation and other aspects of growth and development. Plant Cell 9:1973-1983.

Choi, Y. E., and Xu, J. R. 2010. The cAMP signaling pathway in Fusarium verticillioides is important for conidiation, plant infection, and stress responses but not fumonisin production. Mol. Plant-Microbe Interact. 23:522-533.

Cuomo, C. A., Gueldener, U., Xu, J. R., Trail, F., Turgeon, B. G., Di Pietro, A., Walton, J. D., Ma, L. J., Baker, S. E., Rep, M., Adam, G., Antoniw, J., Baldwin, T., Calvo, S., Chang, Y. L., Decaprio, D., Gale, L. R., Gnerre, S., Goswami, R. S., Hammond-Kosack, K., Harris, L. J., Hilburn, K., Kennell, J. C., Kroken, S., Magnuson, J. K., Mannhaupt, G., Mauceli, E., Mewes, H. W., Mitterbauer, R., Muehlbauer, G., Münsterkötter, M., Nelson, D., O’Donnell, K., Ouellet, T., Qi, W., Quesneville, H., Roncero, M. I., Seong, K. Y., Tetko, I. V., Urban, M., Waalwijk, C., Ward, T. J., Yao, J., Birren, B. W., and Kistler, H. C. 2007. The Fusarium graminearum genome reveals a link between localized polymorphism and pathogen specialization. Science 317:14001402.

Desjardins, A. E. 2003. Gibberella from A (venaceae) to Z (eae). Annu. Rev. Phytopathol. 41:177-198.

Ding, S. L., Mehrabi, R., Koten, C., Kang, Z. S., Wei, Y. D., Seong, K. Y., Kistler, H. C., and Xu, J. R. 2009. Transducin beta-like gene FTL1 is essential for pathogenesis in Fusarium graminearum. Eukaryot. Cell 8:867-876.

D'Souza, C. A., and Heitman, J. 2001. Conserved cAMP signaling cascades regulate fungal development and virulence. FEMS (Fed. Eur. Microbiol. Soc.) Microbiol. Rev. 25:349-364.

Durrenberger, F., Wong, K., and Kronstad, J. W. 1998. Identification of a cAMP-dependent protein kinase catalytic subunit required for virulence and morphogenesis in Ustilago maydis. Proc. Natl. Acad. Sci. U.S.A. 95:5684-5689.

Fuller, K. K., and Rhodes, J. C. 2012. Protein kinase A and fungal virulence A sinister side to a conserved nutrient sensing pathway. Virulence 3:109-121.

Gale, L. R., Ward, T. J., Balmas, V., and Kistler, H. C. 2007. Population subdivision of Fusarium graminearum sensu stricto in the upper Midwestern United States. Phytopathology 97:1434-1439.

Gardiner, D. M., Kazan, K., and Manners, J. M. 2009. Novel genes of Fusarium graminearum that negatively regulate deoxynivalenol production and virulence. Mol. Plant-Microbe Interact. 22:1588-1600.

Goswami, R. S., and Kistler, H. C. 2004. Heading for disaster: Fusarium graminearum on cereal crops. Mol. Plant Pathol. 5:515-525.

Hou, Z. M., Xue, C. Y., Peng, Y. L., Katan, T., Kistler, H. C., and Xu, J. R. 2002. A mitogen-activated protein kinase gene (MGV1) in Fusarium graminearum is required for female fertility, heterokaryon formation, and plant infection. Mol. Plant-Microbe Interact. 15:1119-1127.

Kang, Z. S., Buchenauer, H., Huang, L. L., Han, Q. M., and Zhang, H. C. 2008. Cytological and immunocytochemical studies on responses of wheat spikes of the resistant Chinese cv. Sumai 3 and the susceptible cv. Xiaoyan 22 to infection by Fusarium graminearum. Eur. J. Plant Pathol. 120:383-396.

Kim, H. K., and Yun, S. H. 2011. Evaluation of potential reference genes for quantitative RT-PCR analysis in Fusarium graminearum under different culture conditions. Plant Pathol. J. 27:301-309.

Kim, H. S., Park, S. Y., Lee, S., Adams, E. L., Czymmek, K., and Kang, S. 2011. Loss of cAMP-dependent protein kinase A affects multiple traits important for root pathogenesis by Fusarium oxysporum. Mol. PlantMicrobe Interact. 24:719-732.

Kim, J. E., Han, K. H., Jin, J. M., Kim, H., Kim, J. C., Yun, S. H., and Lee, Y. W. 2005. Putative polyketide synthase and laccase genes for biosynthesis of aurofusarin in Gibberella zeae. Appl. Environ. Microbiol. 71:1701-1708.

Kim, J. E., Jin, J. M., Kim, H., Kim, J. C., Yun, S. H., and Lee, Y. W. 2006. GIP2, a putative transcription factor that regulates the aurofusarin biosynthetic gene cluster in Gibberella zeae. Appl. Environ. Microbiol. 72:1645-1652.

Lee, N., D'Souza, C. A., and Kronstad, J. W. 2003. Of smuts, blasts, mildews, and blights: cAMP signaling in phytopathogenic fungi. Annu. 
Rev. Phytopathol. 41:399-427.

Lengeler, K. B., Davidson, R. C., D’Souza, C., Harashima, T., Shen, W. C., Wang, P., Pan, X. W., Waugh, M., and Heitman, J. 2000. Signal transduction cascades regulating fungal development and virulence. Microbiol. Mol. Biol. Rev. 64:746-785.

Li, G. T., Zhou, X. Y., and Xu, J. R. 2012. Genetic control of infectionrelated development in Magnaporthe oryzae. Curr. Opin. Microbiol. 15:678-684.

Liebmann, B., Muller, M., Braun, A., and Brakhage, A. A. 2004. The cyclic AMP-dependent protein kinase A network regulates development and virulence in Aspergillus fumigatus. Infect. Immun. 72:5193-5203.

Lindquist, S., and Craig, E. A. 1988. The heat-shock proteins. Annu. Rev. Genet. 22:631-677.

Livak, K. J., and Schmittgen, T. D. 2001. Analysis of relative gene expression data using real-time quantitative PCR and the 2(T)(-Delta Delta C) method. Methods 25:402-408.

Lysøe, E., Pasquali, M., Breakspear, A., and Kistler, H. C. 2011. The transcription factor FgStuAp influences spore development, pathogenicity, and secondary metabolism in Fusarium graminearum. Mol. PlantMicrobe Interact. 24:54-67.

Mitchell, T. K., and Dean, R. A. 1995. The cAMP-dependent protein kinase catalytic subunit is required for appressorium formation and pathogenesis by the rice blast pathogen Magnaporthe grisea. Plant Cell 7:1869-1878.

Ni, M., Rierson, S., Seo, J. A., and Yu, J. H. 2005. The pkaB gene encoding the secondary protein kinase A catalytic subunit has a synthetic lethal interaction with $p k a A$ and plays overlapping and opposite roles in Aspergillus nidulans. Eukaryot. Cell 4:1465-1476.

Proctor, R. H., Hohn, T. M., and McCormick, S. P. 1995. Reduced virulence of Gibberella zeae caused by disruption of a trichothecene toxin biosynthetic gene. Mol. Plant-Microbe Interact. 8:593-601.

Schumacher, J., Kokkelink, L., Huesmann, C., Jimenez-Teja, D., Collado, I. G., Barakat, R., Tudzynski, P., and Tudzynski, B. 2008. The cAMPdependent signaling pathway and its role in conidial germination, growth, and virulence of the gray mold Botrytis cinerea. Mol. PlantMicrobe Interact. 21:1443-1459.

Seo, J. A., Kim, J. C., Lee, D. H., and Lee, Y. W. 1996. Variation in 8-ketotrichothecenes and zearalenone production by Fusarium graminearum isolates from corn and barley in Korea. Mycopathologia 134:31-37.

Seong, K., Hou, Z. M., Tracy, M., Kistler, H. C., and Xu, J. R. 2005. Random insertional mutagenesis identifies genes associated with virulence in the wheat scab fungus Fusarium graminearum. Phytopathology 95:744-750.

Shimizu, K., and Keller, N. P. 2001. Genetic involvement of a cAMPdependent protein kinase in a $\mathrm{g}$ protein signaling pathway regulating morphological and chemical transitions in Aspergillus nidulans. Genetics 157:591-600.

Toda, T., Cameron, S., Sass, P., Zoller, M., and Wigler, M. 1987. Three different genes in $S$. cerevisiae encode the catalytic subunits of the cAMP- dependent protein kinase. Cell 50:277-287.

Tzima, A., Paplomatas, E. J., Rauyaree, P., and Kang, S. 2010. Roles of the catalytic subunit of cAMP-dependent protein kinase A in virulence and development of the soilborne plant pathogen Verticillium dahliae. Fungal Genet. Biol. 47:406-415.

Wang, C. F., Zhang, S. J., Hou, R., Zhao, Z. T., Zheng, Q., Xu, Q. J., Zheng, D. W., Wang, G. H., Liu, H. Q., Gao, X. L., Ma, J. W., Kistler, H. C., Kang, Z. S., and Xu, J. R. 2011. Functional analysis of the kinome of the wheat scab fungus Fusarium graminearum. PLoS Pathog. 7:e1002460. Published online.

Wanjiru, W. M., Kang, Z. S., and Buchenauer, H. 2002. Importance of cell wall degrading enzymes produced by Fusarium graminearum during infection of wheat heads. Eur. J. Plant Pathol. 108:803-810.

Xu, H. J., Wang, Y. J., Zhao, P. B., Zhang, Y. B., Xu, R. Y., and Li, D. C. 2011. A cAMP-dependent protein kinase gene, aapk1, is required for mycelia growth, toxicity and pathogenicity of Alternaria alternata on Tobacco. J. Phytopathol. 159:208-216.

Yamauchi, J., Takayanagi, N., Komeda, K., Takano, Y., and Okuno, T. 2004. cAMP-PKA signaling regulates multiple steps of fungal infection cooperatively with Cmk1 MAP kinase in Colletotrichum lagenarium. Mol. Plant-Microbe Interact. 17:1355-1365.

Yang, Z. H., and Dickman, M. B. 1999. Colletotrichum trifolii mutants disrupted in the catalytic subunit of cAMP-dependent protein kinase are nonpathogenic. Mol. Plant-Microbe Interact. 12:430-439.

Zheng, D., Zhang, S., Zhou, X., Wang, C., Xiang, P., Zheng, Q., and Xu, J.-R. 2012a. The FgHOG1 Pathway Regulates Hyphal Growth, Stress Responses, and Plant Infection in Fusarium graminearum. PLoS One 7:e49495. Published online.

Zheng, Q., Hou, R., Zhang, J., Ma, J., Wu, Z., Wang, G., Wang, C., and $\mathrm{Xu}$, J.-R. 2013. The MAT locus genes play different roles in sexual reproduction and pathogenesis in Fusarium graminearum. PLoS One 8:e66980. Published online.

Zheng, W. H., Zhao, X., Xie, Q. R., Huang, Q. P., Zhang, C. K., Zhai, H. C., Xu, L. P., Lu, G. D., Shim, W. B., and Wang, Z. H. 2012b. A conserved homeobox transcription factor $\mathrm{Htf1}$ is required for phialide development and conidiogenesis in Fusarium Species. PLoS One 7:e45432. Published online.

Zhou, X. Y., Heyer, C., Choi, Y. E., Mehrabi, R., and Xu, J. R. 2010. The CID1 cyclin C-like gene is important for plant infection in Fusarium graminearum. Fungal Genet. Biol. 47:143-151.

Zhou, X. Y., Liu, W. D., Wang, C. F., Xu, Q. J., Wang, Y., Ding, S. L., and $\mathrm{Xu}, \mathrm{J}$. R. 2011. A MADS-box transcription factor MoMcm1 is required for male fertility, microconidium production and virulence in Magnaporthe oryzae. Mol. Microbiol. 80:33-53.

Zhou, Z. Z., Li, G. H., Lin, C. H., and He, C. Z. 2009. Conidiophore stalkless 1 encodes a putative Zinc-finger protein involved in the early stage of conidiation and mycelial infection in Magnaporthe oryzae. Mol. Plant-Microbe Interact. 22:402-410. 